\title{
Corona Virus: A Pandemic 2019
}

\author{
Roshani Ramesh Wankhade \\ Medical Intern, Jawaharlal Nehru Medical College, Datta Meghe Institute of Medical Sciences, Wardha \\ roshaniwankhade94@gmail.com
}

Review Article

Conflict of Interest: None

\begin{abstract}
In DECEMBER 2019, the Places for Infectious prevention and Avoidance started to screen the episode of another Covid, SARS-CoV-2, which leads to causes respiratory ailment currently called as Coronavirus - 19 . They previously recognized infection in Wuhan, many cases were reported in China seafood market . In excess of 82542 instance of Covid in China upto 31 Walk 2020. Wellbeing specialists had recognized numerous others with Coronavirus - 19 around the globe. On 31 Walk 2020, the infection spread among in excess of 750890 individuals all through the World. The World Wellbeing Association (WHO) has pronounced it as a general wellbeing crisis identifying with Coronavirus. Various strains of coronavirus has been analyzed in a few inhabitants of WORLD Population. The CDC had prompted that it is probably going to Affect many countries all over the world. Coronavirus influenced numerous nations on the planet. The primary individuals with Coronavirus had connections to a creature and fish market. This reality proposed that creatures at first communicated the infection to people. Nonetheless, individuals with a later finding had no associations with or openness to the market, affirming that people can pass the infection to one another. Covid will taint the vast majority sooner or later during their lifetime. Covid can change successfully, which makes them so infectious. Data on the infection is scant as of now. On 17 February 2020, the Chief General of the WHO introduced at a media preparation that accompanying reports on how regularly this manifestations of Coronavirus . However, some infections are exceptionally infectious, it is now clear that how quickly Coronavirus is spreading throughout the globe. Indicationsof this disease changes from person to person according to their immune response. In some this might deliver not many or no manifestations depending on many factors like age, associated underlying conditions, etc. Be that as it may, it can likewise prompt extreme ailment and might be deadly. On 11 Walk 2020, WHO proclaimed Novel Covid Sickness (Coronavirus) episode as a Pandemic.
\end{abstract}

Keywords: Corona, Covid, Pandemic, SARS, Respiratory

\section{INTRODUCTION}

In finish of Nov. and beginning of December 2019, hardly any instance of obscure Respiratory Contamination was accounted for in Wuhan and Hubei, China. By 31 walk 2019 WHO country office in china was reported with outbreak of pneumonia of unknown origin after that centre for disease control and prevention begins to monitor all new cases Its all signs and symptoms are same as that of viral pneumonia. After examination on respiratory examples, PRC Communities for Infectious prevention specialists proclaimed that the pneumonia, later known as novel coronavirus (coronavirus), was brought about by that of crown virus[1]. 30 dec 2019 WHO officially declared novel corona virus as it public health emergency of international health concern. COVID-19 is name given by

WHO the Coronavirus is caused by the different strains of Coronavirus. 'CO' means for CORONA, 'VI' stands for VIRUS , and 'D' stands for DISEASEand 19 refer to as 2019 year because it was started during this year. Earlier, this sickness was alluded to as '2019 novel Covid's or '2019-nCoV'. Coronavirus is a large family of viruses causing illness to human being as well as animals throughout the globe .Global Council on Scientific categorization of Infections (ICTV) named this infection extreme most dangerous respiratory tract disorder Coronavirus (SARS-CoV-2). [1]On 11 
Walk 2020, WHO proclaimed Novel Covid Illness (Coronavirus) flare-up as a pandemic and emphasized the call for nations to make quick moves and scale up reaction to treat, identify and lessen the transmission to save individuals' lives. This infection has a place with $\beta$-Covid, an enormous class of infections common in nature.[2][3] Like other infections, Coronavirus has numerous expected normal host, Middle host and last host. This postures extraordinary difficulties to counteraction and treatment of infection contamination. This infection has high contagiousness and infectivity, yet low death rate Contrasted and SARS and MERS,2. Genome examination of novel Covid successions uncovered that the totalgenome arrangement acknowledgment paces of Coronavirus and bat SARS Covid (SARSr-CoVRaTG13) were 79\% and 95\% respectively. It is believed that this coronavirus may start from bat. 29 February 2020, information distributed by World Wellbeing Association indicated that, since 31 Walk 2020 when the principal case was accounted for, 750890 cases were Worldwide affirmed to be tainted by novel Covid and 36405 people were passings in total. Meanwhile, 1071 cases were affirmed, 29 were kicked the bucket in India.[2] It represented an extraordinary danger to worldwide general wellbeing. This report surveys the hereditary structure, contamination source, transmission SARS-CoV-2, so it can give references to subsequent exploration, and may assist perusers with having the most recent comprehension of this new irresistible sickness.

Coronavirus SARS COV 2 the complete infective form of virus outside the host cell is about one twenty five nanometer diametrically and twenty six to thirty two $\mathrm{KB}$ is the range of its genome, the largest of all RNA viruses . Spike(S), envelope (E), and nucleocapsid (A) and are the four structurer proteins [3]
- E protein has no clear function although viral release might be added

- The external surface of the envelop project $M$ protein and it is most important for the viral assembly, which spans the envelope three times .

- The $\mathrm{S}$ protein is the club shaped surface protein giving the virus it's crown like appearance ,characteristically on electron microscopy.

- Receptor binding and fusion with the host cell membrane are functions of $\mathrm{S}$ protein .

SARS COV 2 had evolved into two separate genotypes, which is being said by a an analysis done on population genetics done in January 2020:[3]

- L type: more aggressive and more prevalent (approx. seventy percent of cases in early stages of outbreak :prevalence has been decreased since then)

- S type :less aggressive ,less common and evolutionarily older (around thirty percent of cases)

Coronaviruses are transmitted from animals to humans i.e, are zoonotic .

According to data the horseshoe bats are the natural reservoir of SARS Cov-2 since the genome is identical to bat coronavirus transmission of the coronavirus through the respiratory droplets or aerosolized particles from the mucous membrane of the infected patients to the individuals who come in direct contact.

In the air, within three feet of the infected person larger droplets drop towards the ground while smaller droplets can travel over the six feet and viable for upto three hours under certain conditions .[3]

Droplet or contact transmission is transmission of infectious particles from infected persons to uninfected host through droplets which are more than five micrometer diametrically, either via the touching of the contaminated surface or the contact . 
Airborne transmission differs in that it includes small respiratory droplets known as droplet nuclei that remain hung in the air for longer duration of the time. negating the need for individual contact . $[4,5]$. The historical evidence of human Covid arise in nineteen sixty five when Tyrrell and Bynoe6 come to know that they could emerge as an infection named B-814. It was habitual in human underdeveloped tracheobronchial organ system come from the respiratory lot of a cultivated with a usual virus. The presence of an resistant specialist was exhibited by immunizing the culture from these colonies in trans-nasally in human beings ; colds were delivered in a critical extent of patient, however Tyrrell and Bynoe couldn't cultivate the specialist in tissue media surrounding them. in a similar time line, Hamre and Procknow7 had the choice to cultivate an infection with bizarre characteristics in tissue media from tests got from clinical studies with colds[5]. Both B814 and Hamre's infection, which she called 229E, were ether-touchy and thusly apparently aquired a lipidcontaining coat for infectivity, yet these 2 infections were not recognized with any known myxo-or paramyxoviruses. While practicing in the laboratory of Robert Chanock at the Public Establishments of Wellbeing, McIntosh et al8 published the recovery from illness of different strains of ether-touchy specialists from the human being respiratory lot by using a method similar to that of Tyrrell and Bynoe. These infections were recognized "OC" to recognize that they were filled in organ cavities. In 2019, the Communities for Infectious prevention and Anticipation (CDC) began checking the flare-up of another Covid, SARS-CoV-2, which leads to the respiratory sickness currently called as Coronavirus.[5] Specialists initially distinguished the infection in Wuhan, China. From that point forward, the infection has spread to various nations, throughout the globe driving the World
Wellbeing Association (WHO) to proclaim this as a pandemic. As of April 14, in excess of 2,000,728 individuals have gotten the infection around the world, causing more than $1,26,776$ passings and $4,84,781$ recuperated from Covid. In the India, the infection has influenced more than 11,555 individuals, bringing about in excess of 396 passings.[6] The principal persons withCoronavirus had connections to a creature and fish market. This reality recommended that creatures at first sent the infection to people. In any case, individuals with a later finding had no associations with or openness to the market, affirming that people can pass the infection to one another. Peruse more about how pangolins could be the wellspring of Coronavirus. Data on the infection is scant as of now. Previously, respiratory conditions that create from Covid, for example, SARS and MERS, have spread through close contacts. On Walk 11, 2020, the Chief General of the WHO introduced at a media instructions the accompanying reports on how regularly the side effects of Coronavirus are serious or lethal, The WHO reports that the two gatherings most in danger of encountering extreme disease because of a SARSCoV-2 contamination are more seasoned grown-ups, characterized as "more than 60 years of age", and people who have other medical issue that bargain their safe framework. As indicated by the $\mathrm{CDC}$, kids are not at higher danger for Coronavirus than grown-ups. While there are as of now no distributed logical reports about the helplessness of pregnant ladies, the CDC takes note of that: "Pregnant ladies experience immunologic and physiologic changes which may make them more vulnerable to viral respiratory diseases, including Coronavirus." The CDC additionally prescribe that babies destined to moms with suspected or affirmed Coronavirus are set in segregation as a "individual under scrutiny .[6] 
Various kinds of human Coronavirus differ in how serious the subsequent sickness becomes, and how far they can spread. Doctor as of now perceive seven kinds of Covid that can taint people[7]. Regular sorts 1. 229E (alpha Covid) 2. NL63 (alpha Covid) 3. OC43 (beta Covid) 4. HKU1 (beta Covid). More uncommon strains that cause more extreme confusions incorporate MERS-CoV, which causes Center East respiratory condition [6]. General indications of coronavirus Cold-or influenza like manifestations normally set in from 2-4 days after a Covid disease and are commonly gentle. symptoms ranging from mild symptoms to severe illness that may appears 2-14days after the exposure to the virus . Be that as it may, indications shift from individual to-individual, and a few types of the infection can be deadly[8].

\section{Manifestations may include:}

In the event that you are contaminated with Coronavirus, side effects can be seen between 2- 14 days. It fluctuates from individual to individual.[8]Following are the most well-known indications.

- Absence of appetite(42-84\%)

- Runny nose

- Fatigue(44-70\%)

- Hack (59-82\%)

- Fever(83-99\%)

- Sore throat

- Exacerbated asthma (31-40\%)

- Body hurts (11-35\%)

- Difficulty in breath

- Migrain

- New onset anosmiaand hypojeusia

- Nausea or vomiting

- Lose motions

- Bodily fluid/mucus (28-33\%)

THE LIST OF SYMPTOMPS MAY INCLUDE :

- $\quad$ Congested nose

- $\quad$ Dysetry

- $\quad$ Sputum, coughed up mucus and saliva

- $\quad$ Nausea
- $\quad$ Projectile Vomiting

- Abdominal pain

IF ANYBODY

SHOWING

FOLLOWING SIGNS AND SYMPTOMS THEY MAY SEEK EMERGENCY MEDICAL CARE IMMEDIATELY : [9]

- Difficulty in breathing

- Constant pain or pressure in the chest Confusion state (delirium)

- Inability to wake or stay awake which is also known as narcolepsy

- Cyanosed lips or face

Researchers can only with significant effort develop human Covid in the lab dissimilar to the rhinovirus, which is another reason for the basic virus. This makes it hard to check the effect of the Covid on public economies and general wellbeing. There is no remedy for Covid that cause manifestations looking like the basic virus. Medicines incorporate selfcare and over-the-counter (OTC) drug.

Individuals can make a few strides, including:

- Resting and dodging overexertion

- Drinking a lot of water

- Abstaining From smoking and smoky surrounding

- Taking vitamin $\mathrm{c}$ and vitamin $\mathrm{b}$ rich fruits

- Dailly consumption of fruits and vegetables

- Cool fog vaporizer A specialist analyze the infection mindful by considering an example of respiratory fluids, for example, bodily fluid from the nose or pharynx , or blood. [9]

\section{DISCUSSION:}

In DECEMBER 2019, the Places for Infectious prevention and Avoidance started to screen the episode of another Covid, SARS-CoV-2, which leads to causes respiratory ailment currently called as Coronavirus - 19 . They previously recognized infection in Wuhan,many cases were reported in China seafood market . In excess of 82542 instance of Covid in China upto 31 Walk 2020. Wellbeing 
specialists had recognized numerous others with Coronavirus - 19 around the globe. On 31 Walk 2020, the infection spread among in excess of 750890 individuals all through the World.[10] The World Wellbeing Association (WHO) has pronounced it as a general wellbeing crisis identifying with Coronavirus. Covid have a place with the subfamily Coronavirinae in the family Coronaviridae. Various sorts of human Covid differ in how serious the subsequent illness becomes, and how far they can spread. Specialists at present perceive seven sorts of Covid that can contaminate people.[10]

Normal sorts include:

- 229E (alpha Covid)

- NL63 (alpha Covid)

- OC43 (beta Covid)

- HKU1 (beta Covid)

More uncommon strains which leads to more serious confusions incorporate MERS-CoV, which may result in (MERS), and SARS-CoV, the infection liable for more extreme intense respiratory condition (SARS).

Restricted exploration is accessible on how $\mathrm{HCoV}$ spreads starting with one individual then onto the next. In any case, analysts accept that the infections send through liquids in the respiratory framework, for example, bodily fluid.

Covid can spread in the accompanying manners:

- Coughing and sneezing without covering the mouth can scatter beads into the air

- Contacting or warmly greeting an individual who has the infection can pass the infection between people.

- Connecting with a surface or article that has the infection and afterward contacting the nose, eyes, or mouth.

- Some creature Covid, for example, cat Covid (FCoV), may spread through contact with defecation. Notwithstanding, it is indistinct whether this likewise applies to human Covid.
- Covid will contaminate a great many people eventually during their lifetime.

- Covid can transform viably, which makes them so infectious.

To forestall transmission, individuals should remain at home and rest while indications are dynamic. They ought to likewise maintain a strategic distance from close contact with others. Infection spreads from individual to individual . it's critical to restrict your contact with others however much as could reasonably be expected. A few people work in "fundamental organizations" that are indispensable to everyday life, for example, medical care, law requirement, and public utilities. Every other person should remain at home as much as possible. You may hear authorities utilize these terms when they talk about remaining at home. Covering the mouth when wheezing may help stop the spread of Covid. BY taking precautions like covering the mouth and nose with a tissue or hanky while hacking or sniffling can likewise helpful to reduce the forestall transmission. It is really important to discard any tissues after utilize and maintain the cleanliness around the home (surrounding ). [10]

\section{MANAGEMENT:}

There is no specific treatment for coronavirus available right now, and there is no pre and post prophylaxis is available nor recommended. According to the condition of individual it can be managed as follows it will be treated according to the condition such as MILD, MODERATE AND SEVERE . By diagnosing the condition of the patient it can be treated as follows:

Incubation period for the coronavirus is 214 days average 5 days and are commonly gentle .[11,12]

General indications of coronavirus cold or influenza like manifestations normally set in from 2-14 days after the disease . 
Mild patients can be treated by giving them symptomatic treatment by counselling the patient according to their sign and symptoms such as antipyretics for fever and pain, vitamin c for boosting immunity, zinc table( which are showing good results by stopping the viral replication mostly ) vitamin b2, b4 and b6 and adequate nutrition and proper rest . Isolation of the patient plays important role to stopped the spread of coronavirus [13-16].

Moderate patient can be treated with same protocol which is given for the mild Patient it may not require emergency intervention or hospitalization . Patient must be isolated for the specific duration of time . for the patients which are at higher risk may prefer hospitalization, as recommended monitoring patients vitals is necessary oxygen levels fluctuates mostly in this cases.To avoid any risk it is important to maintain the oxygen saturation of the patient above 90 [17-20].

Severe patient most important is ICCU care is must for closely monitoring the patient for signs of deterioration of patients condition this condition is mostly affect the respiratory tract of the Patient .Fluid management in coronavirus patient without tissue hypoperfusion and responsiveness.

\section{Coronavirus in children $[21,22]$}

Coronavirus in children may different from that of adults. Young adults are at lower risk of developing this disease. Development severe or critical infections , and complications may appears milder in children .

Approximately fifty five percent of cases are asymptomatic and mild, forty percent cases are with pneumonia and also with abnormal chest imaging in children and five percent with dyspnea requiring oxygen therapy . one percent of the cases are with acute respiratory distress syndrome , shock, many organ failure, requiring emergency ICU transfer of the patient otherwise it may lead serious complications which cannot be treated if not handle carefully .

\section{CONCLUSION :}

Humankind's arrangement of encounters is seeing an amazingly irregular time engaging an imperceptible enemy, over the span of ongoing years the advancement of a wide scope of Covid that cause a wide variety of human diseases has occurred. Taking everything into the account, these contaminations will continue to emerge and to create and also cause human scenes inferable from their ability to recombine, change, and spoil different species and cell types. WHO reported Novel Coronavirus Contamination (Covid) erupt as a pandemic on 11 Walk 2020.[12] Future assessment on Covid will continue exploring various pieces of viral Result, Spread, Life cycle, replication and pathogenesis. This review gives a comprehension into the Covid current situation and addresses a picture of the current status of the workmanship with respect to general prosperity influence, pathophysiology and clinical appearances, examination, case the board, emergency response and availability. Understanding the proclivity of these contaminations to bob between species, to develop illness in another host and to perceive colossal archives of Covid will radically help in our ability to anticipate when and where potential scourges may occur. As bats have all the earmarks of being an immense stockpile for these diseases, it will be interesting to choose how they seem to keep an essential separation from clinically clear infection and become industriously polluted. Countless the non-basic and ruffle proteins encoded by these diseases remain uncharacterized with no known limit, and it will be basic to separate frameworks of action for these proteins similarly as describing their part in viral replication and pathogenesis. These article 
steady for ID of crown calm dependent on Sign and the best practice for the organization and treatment of intriguing cases and quit Spreading of Covid. Inherited structure of Coronavirus.

\section{REFERENCES:}

[1] Huang C, Wang Y, Li X, Ren L, Zhao J, Hu Y, et al. Clinical features of patients infected with 2019 novel corona virus in Wuhan, China. Lancet (London, England). 2020; 395:497-506.

[2] Liu Y, Gayle AA, Wilder-Smith A, Rocklov J. The reproductive number of COVID-19 is higher compared to SARS corona virus. J Travel Med. 2020.

[3] Chen N, Zhou M, Dong X, Qu J, Gong F, Han Y, et al. Epidemiological and clinical characteristics of 99 cases of 2019 novel corona virus pneumonia in Wuhan, China: a descriptive study. Lancet. 2020.

[4] Organization WHO. Corona virus disease 2019(COVID-19) Situation Report-71. 2020.

[5] Union Ministry of Health and Family Welfare Get the data Created with Datawrapper .

[6] Tyrrell DA, Bynoe ML. Cultivation of viruses from a high proportion of patients with colds. Lancet. 1966;1:76-77.

[7] Hamre D, Procknow JJ. A new virus isolated from the human respiratory tract. Proc Soc Exp Biol Med. 1966; 121:190-193.

[8] McIntosh K, Dees JH, Becker WB, Kapikian AZ, Chanock RM. Recovery in tracheal organ cultures of novel viruses from patients with respiratory disease. Proc Natl Acad Sci USA. 1967; 57:933-940.

[9] Almeida JD, Tyrrell DA. The morphology of three previously uncharacterized human respiratory viruses that grow in organ culture. $\mathbf{J}$ Gen Virol. 1967; 1:175-178.

[10] McIntosh K, Becker WB, Chanock RM. Growth in suckling-mouse brain of "IBV-like" viruses from patients with upper respiratory tract disease. Proc Natl Acad Sci USA. 1967; 58:2268-2273.

[11] Witte KH, Tajima M, Easterday BC. Morphologic characteristics and nucleic acid type of transmissible gastroenteritis virus of pigs. Arch Gesamte Virusforsch. 1968; 23:5370.

[12] Tyrrell DA, Almeida JD, Cunningham $\mathrm{CH}$, et al. Coronaviridae. Intervirology. 1975; 5:76-82.

[13] Parveen, S., and S. Jain. "Pathophysiologic Enigma of COVID-19 Pandemic with Clinical Correlates." International Journal of Current Research and Review 12, no. 13 (2020): 33-37. https://doi.org/10.31782/IJCRR.2020 .12136 .

[14] Chakole, S., and V. Jaiswal. "A Review Paper on Pandemic COVID19." International Journal of Research in Pharmaceutical Sciences 11, no. Special Issue 1 (2020): 99499.

https://doi.org/10.26452/ijrps.v11iSP L1.3404.

[15] Chandankhede, K.J., and R.K. Chandankhede. "Pandemic Disease and Infectious Disease in Ayurveda with Special Reference to Covid-19." International Journal of Research in Pharmaceutical Sciences 11, no. Special Issue 1 (2020): 292-96. https://doi.org/10.26452/ijrps.v11iSP L1.2715.

[16] Chaple, J.N. "Ayurveda and Vyadhikshamatwa (Immunity) during COVID 19." International Journal of Research in Pharmaceutical Sciences 11, no. Special Issue 1 (2020): 1351-55. 
https://doi.org/10.26452/ijrps.v11iSP L1.3639.

[17] Chaudhari, B.V., and P.P. Chawle. "Life Lessons of the Pandemic "COVID-19"." International Journal of Research in Pharmaceutical Sciences 11, no. Special Issue 1 (2020): 469-71. https://doi.org/10.26452/ijrps.v11iSP L1.2814.

[18] Juneja, S., S. Dangore-Khasbage, and R.R. Bhowate. "Role of Vitamin $\mathrm{d}$ in Prevention of Corona Virus Infection (Covid-19)." International Journal of Research in Pharmaceutical Sciences 11, no. Special Issue 1 (2020): 407-10. https://doi.org/10.26452/ijrps.v11iSP L1.2737.

[19] Khan, S., S. Quazi, and M. Kaple. "The Demographical and Epidemiological Profile of Coronavirus Disease 2019 (Covid19)- A Review." Journal of Critical Reviews 7, no. 10 (2020): 4-8. https://doi.org/10.31838/jcr.07.10.02.

[20] Kothari, L., S. Wadatkar, R. Taori, P. Bajaj, and D. Agrawal. "Coronavirus: Towards Controlling of the Pandemic-Indian Scenario." International Journal of Research in Pharmaceutical Sciences 11, no. Special Issue 1 (2020): 462-68. https://doi.org/10.26452/ijrps.v11iSP L1.2813.

[21] Lohiya, S.B., S. Damke, and R. Chaudhary. "Coronavirus Disease (COVID) 2019 in Children - A Short Review." International Journal of Current Research and Review 12, no. 17 (2020): 172-77. https://doi.org/10.31782/IJCRR.2020 .121726.

[22] Sonone, A., A. Hande, M. Gawande, and S. Patil. "Sickle Cell Individuals Are Less Vulnerable for Corona Virus Disease 2019-an Enigma." International Journal of Research in Pharmaceutical Sciences 11, no.
Special Issue 1 (2020): 1015-17. https://doi.org/10.26452/ijrps.v11iSP L1.3427. 